\title{
Effect of Fertilizer and Manure on the Growth, Yield and Grain Nutrient Concentration of Boro Rice (Oryza sativa L.) under Different Water Management Practices
}

\author{
M. M. AL Fakhrul Islam ${ }^{1}$, Md. Asaduzzaman Khan ${ }^{1}$, A. S. M. Fazle Bari ${ }^{1}$, M. T. Hosain ${ }^{2}$ \\ and Sabikunnaher ${ }^{3}$ \\ ${ }^{1}$ Department of Soil Science, ${ }^{2}$ Department of Agricultural Botany, Sher-e-Bangla Agricultural \\ University, Dhaka, Bangladesh; ${ }^{3}$ GME Group, Bangladesh \\ *Corresponding author and Email: fbarisau@gmail.com
}

Received: 29 May $2013 \quad$ Accepted: 06 December 2013

\begin{abstract}
The experiment was conducted in the Sher-e-Bangla Agricultural University research farm, Dhaka, Bangladesh during December 2010 to April 2011 to study the effect of fertilizer and manure with different water management on the growth, yield and nutrient concentration of BRRI dhan28. The experiment consisted of 2 factors i.e. irrigation and fertilizer plus manure. There were 2 irrigation levels $\left(\mathrm{I}_{0}=\right.$ Alternate wetting and drying, $\mathrm{I}_{1}=$ Continuous flooding) and 8 fertilizer treatment $\left(\mathrm{T}_{0}\right.$ : control, $\mathrm{T}_{1}$ : $100 \% \mathrm{RDCF},\left(\mathrm{N}_{100} \mathrm{P}_{15} \mathrm{~K}_{45} \mathrm{~S}_{20} \mathrm{Zn}_{2}\right), \mathrm{T}_{2}: 10$ ton cowdung/ha, $\mathrm{T}_{3}: 50 \% \mathrm{RDCF}+5$ ton cowdung/ha, $\mathrm{T}_{4}: 8$ ton poultry manure/ha, $\mathrm{T}_{5}: 50 \% \mathrm{RDCF}+4$ ton poultry manure/ha, $\mathrm{T}_{6}: 10$ ton vermicompost/ha, $\mathrm{T}_{7}: 50 \% \mathrm{RDCF}+5$ ton vermicompost/ha). There were 16 treatment combinations and 3 replications. Irrigation had no significant effect on the yield and yield parameters of BRRI dhan 28. The yield contributing characters and yields were significantly influenced by applied fertilizer and manure. The $\mathrm{T}_{5}(50 \% \mathrm{RDCF}+4$ ton poultry manure/ha) showed the highest effective tillers/hill, plant height, panicle length, 1000 grain wt., grain yield $(5.92 \mathrm{~kg} / \mathrm{plot})$ and straw yield $(5.91 \mathrm{~kg} / \mathrm{plot})$. The higher grain and straw yields were obtained organic manure plus inorganic fertilizers than full dose of chemical fertilizer and manure. The highest grain $(5.93 \mathrm{~kg} / \mathrm{plot})$ and straw yields $(6.42 \mathrm{~kg} / \mathrm{plot})$ were recorded from $\mathrm{I}_{0} \mathrm{~T}_{5}$ (Alternate wetting and drying $+50 \%$ RDCF plus 4 ton poultry manure/ha) and the lowest was found in $\mathrm{I}_{1} \mathrm{~T}_{0}$ (Continuous flooding + control treatment) treatment combination. The highest concentrations of grain and straw $\mathrm{N}, \mathrm{P}, \mathrm{K}, \mathrm{S}$ were recorded in $\mathrm{T}_{5}$ treatment. The levels of organic matter and nutrient concentration were increased in the post harvest soils due to added manure plus inorganic fertilizer.
\end{abstract}

Keywords: Cowdung, poultry manure, vermicompost, NPKS, irrigation, rice, yield

\section{Introduction}

Rice (Oryza sativa L.) is the staple food of Bangladesh. Among the three rice seasons, boro rice covers about $56.66 \%$ of total rice area and it contributes to $43.24 \%$ of the total rice production in the country (BBS, 2008). Rice is intensively cultivated in Bangladesh covering about $80 \%$ of arable land. Unfortunately, the yield of rice is low in Bangladesh than in the other rice growing countries like South Korea and Japan where the average yield is 7.00 and $6.22 \mathrm{t} / \mathrm{ha}$, respectively (FAO, 1999). On the other hand, the demand for increasing rice production is mounting up to feed 
the ever-increasing population. A suitable combination of organic and inorganic sources of nutrients is necessary for sustainable agriculture that can ensure food production with high quality. Applications of both chemical and organic fertilizers need to be applied for the improvement of soil physical properties and supply of essential plant nutrients for higher yield. The application of different levels of irrigation in boro rice affect the yield, nutrient accumulation and boro rice quality. More nutrients are leached out from soil when higher levels of irrigation water are added during boro rice growing period. Moisture levels affect the organic matter accumulation and mineralization. Yang et al. (2004) reported that application of chemical fertilizers with farmyard manure or wheat straw in alternate wetting and drying condition increased $\mathrm{N}, \mathrm{P}, \& \mathrm{~K}$ uptake by rice plants, increased 1000 grain weight and grain yield of rice. Use of appropriate levels of fertilizer and irrigation in boro rice is important for maintaining productivity and fertility.

Considering the present situation the present study was undertaken to: develop a suitable integrated dose of inorganic fertilizers combined with different manures for Boro rice; evaluate the effects of inorganic and organic fertilizer with different water management on the yield, yield components and nutrient concentration of Boro rice; and investigate the improvement of soil fertility due to the use of organic manure in combination with chemical fertilizers.

\section{Materials and Methods}

The research work was conducted at the farm of Sher-e-Bangla Agricultural University, Dhaka 1207 during the Boro season of 2010-2011. The soil of the experimental field belongs to the Tejgaon series of AEZ No. 28, Madhupur Tract, classified as Deep Red Brown Terrace Soils in Bangladesh soil classification system. The soil texture was silt loam having $\mathrm{pH} 6.1$ and contains organic matter $1.09 \%$, total $\mathrm{N} 0.04 \%$, available $\mathrm{P}$ $9.9 \mathrm{ppm}$, available $\mathrm{K} 15.6 \mathrm{ppm}$, and available $\mathrm{S}$ 8.06 ppm. BRRI dhan 28 was used as the test crop in this experiment. The experiment consists of 2 factors i. e. irrigation and fertilizer plus manure. There were 2 irrigation levels $\left(\mathrm{I}_{0}=\right.$ Alternate wetting and drying, $\mathrm{I}_{1}=$ Continuous flooding) and 8 fertilizer treatment $\left(\mathrm{T}_{0}\right.$ : control, $\mathrm{T}_{1}: 100 \%$ RDCF, $\left(\mathrm{N}_{100} \mathrm{P}_{15} \mathrm{~K}_{45} \mathrm{~S}_{20} \mathrm{Zn}_{2}\right), \mathrm{T}_{2}: 10$ ton cowdung/ha, $\mathrm{T}_{3}: 50 \% \mathrm{RDCF}+5$ ton cowdung/ha, $\mathrm{T}_{4}: 8$ ton poultry manure/ha, $\mathrm{T}_{5}$ : $50 \% \mathrm{RDCF}+4$ ton poultry manure/ha, $\mathrm{T}_{6}: 10$ ton vermicompost/ha, $\mathrm{T}_{7}: 50 \% \mathrm{RDCF}+5$ ton vermicompost/ha) to form 16 treatment combinations.

The experiment was laid out in a split plotdesign (SPD) with three replications with the two irrigations levels in the main plots and fertilizer plus manure treatments to the sub plots. The total numbers of plots were 48 . The plot size was $3 \mathrm{~m} \times 3 \mathrm{~m}$ and ailes separated the plots from each other. The distance maintained between two main plots and two-sub plots were $1.0 \mathrm{~m}$ and 0.5 $\mathrm{m}$, respectively. The experimental plot was opened by a tractor, and then the land was ploughed and cross-ploughed several times with the help of a power tiller followed by laddering to obtain a good tilth and puddled condition Weeds and stubbles were removed, and the large clods were broken into smaller pieces to obtain a desirable tilth. Finally, the land was leveled and the experimental plot was partitioned into the unit plots.

Full amounts of TSP, MP, gypsum and zinc sulphate were applied as basal dose before transplanting of rice seedlings. Urea were applied in 3 equal splits: one third was applied at basal before transplanting, one third at active tillering stage (30 DAT) and the remaining one third was applied at 5 days before panicle initiation stage (55 DAT). Cowdung, poultry manures and vermicompost were applied before four days of final land preparation. Forty days old seedlings of BRRI dhan 28 were transplanted on $\mathrm{I}^{\mathrm{st}}$ January, 2011 at two seedlings in a spacing of $20 \mathrm{~cm} \times 20 \mathrm{~cm}$. Intercultural operations were done whenever required. The crop was harvested at full maturity when $80-90 \%$ of the grains were turned into straw color on $20^{\text {th }}$ May, 2011. Ten hills of rice plant were selected randomly from 
the plants for measuring yield contributing characters. After drying, the grain and straw yields were determined. The plants, soil and manure samples were collected, processed and analyzed for $\mathrm{N}, \mathrm{P}, \mathrm{K}$ and $\mathrm{S}$. The $\mathrm{N}$ concentration of cowdung, poultry manure and vermicompost were $1.46,2.2$ and $1.49 \%$ respectively. The available (soil) and total $\mathrm{P}$ (plant) were determined by ascorbic acid blue color method (Olsen et al., 1954). Exchangeable (soil) and total $\mathrm{K}$ (plant) were determined by using flame photometer. The available (soil) and total $\mathrm{S}$ (plant) were analysed by turbidimetric method as described by Hunter (1984). N was determined by Micro-Kjeldahl method. The statistical analysis was done using the Duncan's Multiple Range Test (DMRT) at 5\% level of probability (Gomez and Gomez, 1984).

\section{Results and Discussion}

\subsection{Effects of irrigation, fertilizer and manure on the growth and yield of rice}

Application of irrigation on boro rice cultivation influenced the growth and yield of boro rice. The number of effective tillers/hill, grain and straw yields were not significantly different between two levels of irrigations.

Different treatments of fertilizer and manure showed significant variations in respect of effective tillers/hill, plant height, panicle length, number of filled grain per panicle, grain and straw yields of rice (Table 1).

Among the different fertilizer treatments, $\mathrm{T}_{5}$ $(50 \% \mathrm{RDCF}+4$ ton poultry manure/ha) significantly enhanced the number of effective tillers/hill, plant height, panicle length, number of filled grain/panicle, straw yield and grain yield over control (Table 1). Nayak et al. (2007) reported a significant increase in effective tillers/hill due to application of chemical fertilizer with organic manure. Yang et al. (2004) recorded that 1000-grain weight was increased by the application of chemical fertilizer along with organic manure.

Table 1. Effects of different doses of fertilizer and manure on the yield and yield parameters of rice

\begin{tabular}{cccccccc}
\hline Treatments & $\begin{array}{c}\text { No. of } \\
\text { effective } \\
\text { tillers/hill }\end{array}$ & $\begin{array}{c}\text { Plant } \\
\text { height } \\
(\mathrm{cm})\end{array}$ & $\begin{array}{c}\text { Panicle } \\
\text { length } \\
(\mathrm{cm})\end{array}$ & $\begin{array}{c}\text { No. of filled } \\
\text { grain/ } \\
\text { panicle }\end{array}$ & $\begin{array}{c}1000 \\
\text { grain wt. } \\
(\mathrm{g})\end{array}$ & $\begin{array}{c}\text { Grain } \\
\text { yield } \\
(\mathrm{t} / \mathrm{ha})\end{array}$ & $\begin{array}{c}\text { Straw } \\
\text { yield } \\
(\mathrm{t} / \mathrm{ha})\end{array}$ \\
\hline $\mathrm{T}_{0}$ & $9.70 \mathrm{~b}$ & $77.74 \mathrm{~b}$ & $22.16 \mathrm{~b}$ & $114 \mathrm{~b}$ & 19.83 & $4.13 \mathrm{e}$ & $5.10 \mathrm{~b}$ \\
$\mathrm{~T}_{1}$ & $10.49 \mathrm{ab}$ & $83.00 \mathrm{a}$ & $23.04 \mathrm{a}$ & $135 \mathrm{a}$ & 21.33 & $5.98 \mathrm{bc}$ & $5.76 \mathrm{ab}$ \\
$\mathrm{T}_{2}$ & $10.45 \mathrm{ab}$ & $83.66 \mathrm{a}$ & $22.20 \mathrm{a}$ & $135 \mathrm{a}$ & 19.83 & $5.19 \mathrm{~d}$ & $5.83 \mathrm{ab}$ \\
$\mathrm{T}_{3}$ & $10.43 \mathrm{ab}$ & $81.64 \mathrm{ab}$ & $22.19 \mathrm{a}$ & $132 \mathrm{a}$ & 21.00 & $5.55 \mathrm{~cd}$ & $5.87 \mathrm{ab}$ \\
$\mathrm{T}_{4}$ & $10.91 \mathrm{a}$ & $84.22 \mathrm{a}$ & $22.17 \mathrm{a}$ & $133 \mathrm{a}$ & 20.33 & $6.26 \mathrm{ab}$ & $5.99 \mathrm{a}$ \\
$\mathrm{T}_{5}$ & $11.17 \mathrm{a}$ & $84.72 \mathrm{a}$ & $22.90 \mathrm{a}$ & $136 \mathrm{a}$ & 21.83 & $6.57 \mathrm{a}$ & $6.56 \mathrm{a}$ \\
$\mathrm{T}_{6}$ & $10.43 \mathrm{ab}$ & $84.15 \mathrm{a}$ & $22.74 \mathrm{a}$ & $130 \mathrm{a}$ & 21.50 & $5.78 \mathrm{c}$ & $6.03 \mathrm{a}$ \\
$\mathrm{T}_{7}$ & $11.0 \mathrm{a}$ & $85.11 \mathrm{a}$ & $22.64 \mathrm{a}$ & $130 \mathrm{a}$ & 20.17 & $5.81 \mathrm{c}$ & $5.90 \mathrm{ab}$ \\
\hline $\mathrm{SE}( \pm)$ & 0.30 & 1.29 & 0.34 & 3.74 & $\mathrm{NS}$ & 0.24 & 0.22 \\
\hline
\end{tabular}

In a column figures having same letter do not differ significantly whereas figures with different letter differ significantly by DMRT at $5 \%$ level.

[ $\mathrm{T}_{0}$ : control, $\mathrm{T}_{1}: 100 \%$ RDCF, $\left(\mathrm{N}_{100} \mathrm{P}_{15} \mathrm{~K}_{45} \mathrm{~S}_{20} \mathrm{Zn}_{2}\right), \mathrm{T}_{2}: 10$ ton cowdung/ha, $\mathrm{T}_{3}: 50 \% \mathrm{RDCF}+5$ ton cowdung/ha, $\mathrm{T}_{4}: 8$ ton poultry manure/ha, $\mathrm{T}_{5}: 50 \% \mathrm{RDCF}+4$ ton poultry manure/ha, $\mathrm{T}_{6}: 10$ ton vermicompost/ha, $\mathrm{T}_{7}: 50 \%$ $\mathrm{RDCF}+5$ ton vermicompost/ha] 
The highest grain yield (6.57 t/ha) was obtained from $\mathrm{T}_{5}(50 \% \mathrm{RDCF}+4$ ton poultry manure/ha) and the lowest grain yield $(4.13 \mathrm{t} / \mathrm{ha})$ was found with $\mathrm{T}_{0}$ treatment where no fertilizer was applied. The application of fertilizer increased the grain yield from $26-59 \%$ and the highest grain yield was given by $\mathrm{T}_{5}$, where integrated use of fertilizer and manure was used. The result is in agreement with the findings of Miah et al. (2006). The highest straw yield (6.56 t/ha) was recorded in $\mathrm{T}_{5}$ treatment which was similar (6.03 t/ha) to $T_{6}$ treatment and the lowest straw yield (5.10 t/ha) was recorded in the $\mathrm{T}_{0}$ treatment where no fertilizer was applied. Rahman et al. (2009) reported that the application of organic manure and chemical fertilizers increased the grain and straw yields of rice. It is clear that organic manure in combination with inorganic fertilizers increased the vegetative growth of plants and thereby increased straw yield of rice.

\subsection{Interaction effect of fertilizer and irrigation on the grain and straw yield of rice}

The combined effect of different levels of fertilizer and irrigation on the grain and straw yield of rice was significantly different (Table 2). The highest grain yield of rice $(6.58 \mathrm{t} / \mathrm{ha})$ was recorded with the treatment combination $\mathrm{I}_{0} \mathrm{~T}_{5}$ (Alternate wetting and drying $+50 \%$ RDCF plus 4 ton poultry manure/ha) which was statistically similar to $\mathrm{I}_{1} \mathrm{~T}_{5}$ treatment combinations. On the other hand, the lowest grain yield (4.04 t/ha) was found in $\mathrm{I}_{1} \mathrm{~T}_{0}$ (Alternate wetting and drying + control treatment) treatment combination. Similarly, the highest straw yield of rice (7.12 t/ha) was recorded with the treatment combination $\mathrm{I}_{0} \mathrm{~T}_{5}$ and the lowest straw yield (5.07 t/ha) was found in $\mathrm{I}_{1} \mathrm{~T}_{0}$ (Continuous flooding + control treatment) treatment combination. Lin et al. (2011) reported that irrigation with organic material application increased yield of rice.

Table 2. Interaction effect of fertilizer and irrigation on the straw and grain yield of rice

\begin{tabular}{ccc}
\hline Treatments & Grain yield (t/ha) & Straw yield (t/ha) \\
\hline $\mathrm{I}_{0} \mathrm{~T}_{0}$ & $4.21 \mathrm{gh}$ & $5.13 \mathrm{~b}$ \\
$\mathrm{I}_{0} \mathrm{~T}_{1}$ & $5.73 \mathrm{bcde}$ & $5.22 \mathrm{~b}$ \\
$\mathrm{I}_{0} \mathrm{~T}_{2}$ & $4.77 \mathrm{fg}$ & $5.40 \mathrm{~b}$ \\
$\mathrm{I}_{0} \mathrm{~T}_{3}$ & $5.27 \mathrm{ef}$ & $5.74 \mathrm{~b}$ \\
$\mathrm{I}_{0} \mathrm{~T}_{4}$ & $6.33 \mathrm{ab}$ & $5.93 \mathrm{ab}$ \\
$\mathrm{I}_{0} \mathrm{~T}_{5}$ & $6.58 \mathrm{a}$ & $7.12 \mathrm{a}$ \\
$\mathrm{I}_{0} \mathrm{~T}_{6}$ & $5.70 \mathrm{bcde}$ & $6.07 \mathrm{ab}$ \\
$\mathrm{I}_{0} \mathrm{~T}_{7}$ & $5.61 \mathrm{cde}$ & $6.27 \mathrm{ab}$ \\
$\mathrm{I}_{1} \mathrm{~T}_{0}$ & $4.04 \mathrm{~h}$ & $5.07 \mathrm{ab}$ \\
$\mathrm{I}_{1} \mathrm{~T}_{1}$ & $6.25 \mathrm{abc}$ & $6.29 \mathrm{ab}$ \\
$\mathrm{I}_{1} \mathrm{~T}_{2}$ & $5.60 \mathrm{dc}$ & $6.24 \mathrm{ab}$ \\
$\mathrm{I}_{1} \mathrm{~T}_{3}$ & $5.82 \mathrm{bcde}$ & $6.01 \mathrm{ab}$ \\
$\mathrm{I}_{1} \mathrm{~T}_{4}$ & $6.19 \mathrm{abcd}$ & $6.04 \mathrm{ab}$ \\
$\mathrm{I}_{1} \mathrm{~T}_{5}$ & $6.56 \mathrm{a}$ & $5.99 \mathrm{ab}$ \\
$\mathrm{I}_{1} \mathrm{~T}_{6}$ & $5.86 \mathrm{bcde}$ & $6.00 \mathrm{ab}$ \\
$\mathrm{I}_{1} \mathrm{~T}_{7}$ & $5.98 \mathrm{abcd}$ & $5.55 \mathrm{~b}$ \\
\hline $\mathrm{SE}( \pm){ }$ & 0.16 & 0.31 \\
\hline
\end{tabular}

In a column figures having same letter(s) do not differ significantly whereas figures with different letter(s) differ significantly by DMRT at $5 \%$ level.

$\left[\mathrm{I}_{0}=\right.$ Alternate wetting and drying, $\mathrm{I}_{1}=$ Continuous flooding, $\mathrm{T}_{0}$ : control, $\mathrm{T}_{1}: 100 \% \mathrm{RDCF},\left(\mathrm{N}_{100} \mathrm{P}_{15} \mathrm{~K}_{45} \mathrm{~S}_{20} \mathrm{Zn}_{2}\right), \mathrm{T}_{2}$ : 10 ton cowdung/ha, $\mathrm{T}_{3}: 50 \% \mathrm{RDCF}+5$ ton cowdung/ha, $\mathrm{T}_{4}: 8$ ton poultry manure/ha, $\mathrm{T}_{5}: 50 \% \mathrm{RDCF}+4$ ton poultry manure/ha, $\mathrm{T}_{6}: 10$ ton vermicompost/ha, $\mathrm{T}_{7}: 50 \% \mathrm{RDCF}+5$ ton vermicompost/ha] 
Table 3. Effect of fertilizer and manure on NPKS concentration in grain and straw of Boro rice

\begin{tabular}{|c|c|c|c|c|c|c|c|c|}
\hline \multirow{2}{*}{$\begin{array}{c}\text { Treatm } \\
\text { ents }\end{array}$} & \multicolumn{4}{|c|}{ Concentration (\%) in grain } & \multicolumn{4}{|c|}{ Concentration (\%) in straw } \\
\hline & $\mathrm{N}$ & $\mathrm{P}$ & $\mathrm{K}$ & S & $\mathrm{N}$ & $\mathrm{P}$ & $\mathrm{K}$ & $\mathrm{S}$ \\
\hline $\mathrm{T}_{0}$ & $0.833 \mathrm{c}$ & $0.201 \mathrm{e}$ & $0.237 \mathrm{e}$ & $0.082 \mathrm{~d}$ & $0.331 \mathrm{f}$ & $0.044 \mathrm{~d}$ & $0.778 \mathrm{e}$ & 0.055 \\
\hline $\mathrm{T}_{1}$ & $1.083 b c$ & $0.243 \mathrm{c}$ & $0.304 \mathrm{~d}$ & $0.103 \mathrm{c}$ & $0.415 \mathrm{~d}$ & $0.072 \mathrm{ab}$ & $1.159 \mathrm{c}$ & 0.068 \\
\hline $\mathrm{T}_{2}$ & $1.042 \mathrm{bc}$ & $0.226 \mathrm{~d}$ & $0.303 \mathrm{~d}$ & $0.105 \mathrm{bc}$ & $0.402 \mathrm{de}$ & $0.057 \mathrm{~cd}$ & $1.082 \mathrm{~d}$ & 0.066 \\
\hline $\mathrm{T}_{3}$ & $1.020 \mathrm{bc}$ & $0.253 \mathrm{bc}$ & $0.337 \mathrm{~b}$ & $0.118 \mathrm{ab}$ & $0.499 \mathrm{~b}$ & $0.069 \mathrm{abc}$ & $1.097 \mathrm{~d}$ & 0.086 \\
\hline $\mathrm{T}_{4}$ & $1.350 \mathrm{a}$ & $0.255 \mathrm{bc}$ & $0.353 \mathrm{a}$ & $0.126 \mathrm{a}$ & $0.559 \mathrm{a}$ & $0.083 \mathrm{a}$ & $1.286 \mathrm{~b}$ & 0.090 \\
\hline $\mathrm{T}_{5}$ & $1.392 \mathrm{a}$ & $0.280 \mathrm{a}$ & $0.356 \mathrm{a}$ & $0.128 \mathrm{a}$ & $0.564 \mathrm{a}$ & $0.083 \mathrm{a}$ & $1.456 \mathrm{a}$ & 0.080 \\
\hline $\mathrm{T}_{6}$ & $1.217 \mathrm{ab}$ & $0.211 \mathrm{e}$ & $0.300 \mathrm{~d}$ & $0.105 \mathrm{bc}$ & $0.398 \mathrm{e}$ & $0.066 \mathrm{bc}$ & $1.197 \mathrm{c}$ & 0.065 \\
\hline $\mathrm{T}_{7}$ & $1.167 \mathrm{~b}$ & $0.267 \mathrm{ab}$ & $0.322 \mathrm{c}$ & $0.112 b c$ & $0.477 \mathrm{c}$ & $0.058 \mathrm{bcd}$ & $1.084 \mathrm{~d}$ & 0.071 \\
\hline $\mathrm{SE}( \pm)$ & 0.0732 & 0.0062 & 0.0043 & 0.0021 & 0.0062 & 0.0019 & 0.0127 & NS \\
\hline
\end{tabular}

In a column figures having same letter do not differ significantly whereas figures with different letter differ significantly by DMRT at $5 \%$ level.

[ $\mathrm{T}_{0}$ : control, $\mathrm{T}_{1}: 100 \% \mathrm{RDCF},\left(\mathrm{N}_{100} \mathrm{P}_{15} \mathrm{~K}_{45} \mathrm{~S}_{20} \mathrm{Zn}_{2}\right), \mathrm{T}_{2}: 10$ ton cowdung/ha, $\mathrm{T}_{3}: 50 \% \mathrm{RDCF}+5$ ton cowdung/ha, $\mathrm{T}_{4}: 8$ ton poultry manure/ha, $\mathrm{T}_{5}: 50 \% \mathrm{RDCF}+4$ ton poultry manure/ha, $\mathrm{T}_{6}: 10$ ton vermicompost/ha, $\mathrm{T}_{7}: 50 \%$ $\mathrm{RDCF}+5$ ton vermicompost/ha]

\subsection{Effect of fertilizer and manure on $N, P, K$ and $S$ concentration in grain and straw}

The nitrogen, phosphorus, potassium and sulphur content in grain and straw were significantly influenced with the different levels of fertilizer and manure application (Table 3). The higher levels of $\mathrm{N}, \mathrm{P}, \mathrm{K}$ and $\mathrm{S}$ concentrations in grain and straw were recorded in the combined application of fertilizer and manure compared to the chemical fertilizer alone.

The grain and straw of $\mathrm{T}_{5}(50 \% \mathrm{RDCF}+4$ ton poultry manure/ha) treatment contained the highest levels of $\mathrm{N}, \mathrm{P}, \mathrm{K}$ and $\mathrm{S}$ which was closely followed by $\mathrm{T}_{4}$ ( 8 ton poultry manure/ha) treatment (Table 3). On the other hand, the $\mathrm{T}_{0}$ showed the lowest $\mathrm{N}, \mathrm{P}, \mathrm{K}$ and $\mathrm{S}$ concentration in grain and straw. Similar results were also found by investigators Singh et al. (2001)).

\subsection{Effect of fertilizer on $\mathrm{pH}, \mathrm{OM}, \mathrm{N} P \mathrm{~K}$ and $\mathrm{S}$ of post harvest soil}

Different irrigations had no significant change on $\mathrm{pH}$, organic matter, $\mathrm{N}, \mathrm{P}, \mathrm{K}$ content in post harvest soils. The application of different fertilizer and manure significantly influenced the $\mathrm{pH}, \mathrm{OM}$ and $\mathrm{N}, \mathrm{P}, \mathrm{K}, \mathrm{S}$ concentrations of post harvest soil (Table 4). The highest $\mathrm{pH}$ of post harvest soil (6.4) was recorded from $\mathrm{T}_{1}(100 \%$ RDCF, $\left(\mathrm{N}_{100} \mathrm{P}_{15} \mathrm{~K}_{45} \mathrm{~S}_{20} \mathrm{Zn}_{2}\right)$ treatment which was comparable to $\mathrm{T}_{2} \mathrm{~T}_{4}$ treatments.

The level of organic matter in post harvest soil increased with combined application of fertilizer and manure. The highest organic matter of post harvest soil (1.24\%) was recorded from $\mathrm{T}_{5}(50 \%$ $\mathrm{RDCF}+4$ ton poultry manure/ha) which was followed by $\mathrm{T}_{4}$ ( 8 ton poultry manure/ha) and the lowest organic matter of post harvest soil was $(1.0 \%)$ recorded from $\mathrm{T}_{0}$ (control) treatment. $\mathrm{Xu}$ et al. (2008) reported that application of chemical fertilizer with organic manure increase soil organic matter. The soil of $T_{2}$ (10 ton cowdung/ha) treatment contained the highest levels of $\mathrm{N}(0.093 \%)$. The highest available $\mathrm{P}$ (34.08 ppm) and $\mathrm{K}(0.042 \mathrm{meq} / 100 \mathrm{~g})$ in post harvest soil was recorded from $\mathrm{T}_{5}$ which was statistically identical to with $\mathrm{T}_{4}$. Ayoola \& Makinde (2009) found that application of chemical fertilizer with organic manure increased N, P, K content in post harvest soil. The highest available sulphur in post harvest soil was (43.39 ppm) recorded from $\mathrm{T}_{3}$ and the lowest NPKS content of post harvest soil was observed from $\mathrm{T}_{0}$ treatment. 
Table 4. Effect of fertilizer and manure on the $\mathrm{pH}$, organic matter and NPKS content in post harvest soil

\begin{tabular}{ccccccc}
\hline $\begin{array}{c}\text { Treatme } \\
\text { nts }\end{array}$ & $\mathrm{pH}$ & $\begin{array}{c}\text { Organic } \\
\text { matter }(\%)\end{array}$ & $\begin{array}{c}\text { Total N } \\
(\%)\end{array}$ & $\begin{array}{c}\text { Available P } \\
(\mathrm{ppm})\end{array}$ & $\begin{array}{c}\text { Available K } \\
(\mathrm{meq} / 100 \mathrm{~g})\end{array}$ & $\begin{array}{c}\text { Available } \mathrm{S} \\
(\mathrm{ppm})\end{array}$ \\
\hline $\mathrm{T}_{0}$ & $6.1 \mathrm{ab}$ & $1.0 \mathrm{~h}$ & 0.054 & $19.95 \mathrm{e}$ & $0.020 \mathrm{c}$ & $21.395 \mathrm{c}$ \\
$\mathrm{T}_{1}$ & $6.4 \mathrm{a}$ & $1.06 \mathrm{~g}$ & 0.091 & $26.03 \mathrm{~d}$ & $0.039 \mathrm{ab}$ & $29.755 \mathrm{abc}$ \\
$\mathrm{T}_{2}$ & $6.3 \mathrm{ab}$ & $1.13 \mathrm{f}$ & 0.093 & $31.89 \mathrm{ab}$ & $0.032 \mathrm{abc}$ & $33.232 \mathrm{abc}$ \\
$\mathrm{T}_{3}$ & $6.0 \mathrm{~b}$ & $1.16 \mathrm{~d}$ & 0.067 & $27.93 \mathrm{~cd}$ & $0.037 \mathrm{ab}$ & $43.388 \mathrm{a}$ \\
$\mathrm{T}_{4}$ & $6.2 \mathrm{ab}$ & $1.22 \mathrm{~b}$ & 0.062 & $32.14 \mathrm{ab}$ & $0.042 \mathrm{a}$ & $34.432 \mathrm{abc}$ \\
$\mathrm{T}_{5}$ & $6.1 \mathrm{ab}$ & $1.24 \mathrm{a}$ & 0.089 & $34.08 \mathrm{a}$ & $0.042 \mathrm{a}$ & $40.755 \mathrm{ab}$ \\
$\mathrm{T}_{6}$ & $6.1 \mathrm{ab}$ & $1.16 \mathrm{e}$ & 0.072 & $29.29 \mathrm{bcd}$ & $0.026 \mathrm{bc}$ & $27.873 \mathrm{bc}$ \\
$\mathrm{T}_{7}$ & $6.0 \mathrm{~b}$ & $1.17 \mathrm{c}$ & 0.078 & $30.49 \mathrm{abc}$ & $0.034 \mathrm{abc}$ & $29.302 \mathrm{bc}$ \\
\hline $\mathrm{SE}( \pm)$ & 0.076 & 0.009 & $\mathrm{NS}$ & 1.015 & 0.005 & 3.842 \\
\hline
\end{tabular}

In a column figures having same letter do not differ significantly whereas figures with different letter differ significantly by DMRT at $5 \%$ level.

[ $\mathrm{T}_{0}$ : control, $\mathrm{T}_{1}: 100 \% \mathrm{RDCF},\left(\mathrm{N}_{100} \mathrm{P}_{15} \mathrm{~K}_{45} \mathrm{~S}_{20} \mathrm{Zn}_{2}\right), \mathrm{T}_{2}: 10$ ton cowdung/ha, $\mathrm{T}_{3}: 50 \% \mathrm{RDCF}+5$ ton cowdung/ha, $\mathrm{T}_{4}: 8$ ton poultry manure/ha, $\mathrm{T}_{5}: 50 \% \mathrm{RDCF}+4$ ton poultry manure/ha, $\mathrm{T}_{6}: 10$ ton vermicompost/ha, $\mathrm{T}_{7}: 50 \%$ $\mathrm{RDCF}+5$ ton vermicompost/ha]

Table 5. Combined effects of fertilizer and irrigation on the $\mathrm{pH}$, organic matter and NPKS concentration of post harvest soil

\begin{tabular}{ccccccc}
\hline Treatments & $\mathrm{pH}$ & $\begin{array}{c}\text { Organic } \\
\text { matter }(\%)\end{array}$ & $\begin{array}{c}\text { Total N } \\
(\%)\end{array}$ & $\begin{array}{c}\text { Available P } \\
(\mathrm{ppm})\end{array}$ & $\begin{array}{c}\text { Available } \\
\mathrm{K}(\mathrm{ppm})\end{array}$ & $\begin{array}{c}\text { Available } \mathrm{S} \\
(\mathrm{ppm})\end{array}$ \\
\hline $\mathrm{I}_{0} \mathrm{~T}_{0}$ & $6.4 \mathrm{abc}$ & $1.01 \mathrm{j}$ & 0.050 & 19.53 & 0.021 & $21.51 \mathrm{~cd}$ \\
$\mathrm{I}_{0} \mathrm{~T}_{1}$ & $6.5 \mathrm{a}$ & $1.00 \mathrm{k}$ & 0.064 & 25.56 & 0.044 & $27.85 \mathrm{bcd}$ \\
$\mathrm{I}_{0} \mathrm{~T}_{2}$ & $6.4 \mathrm{abc}$ & $1.13 \mathrm{~g}$ & 0.064 & 29.87 & 0.022 & $36.90 \mathrm{abc}$ \\
$\mathrm{I}_{0} \mathrm{~T}_{3}$ & $6.3 \mathrm{abcde}$ & $1.17 \mathrm{~d}$ & 0.057 & 25.64 & 0.037 & $31.80 \mathrm{bcd}$ \\
$\mathrm{I}_{0} \mathrm{~T}_{4}$ & $6.3 \mathrm{abcd}$ & $1.22 \mathrm{~b}$ & 0.067 & 29.52 & 0.040 & $28.76 \mathrm{bcd}$ \\
$\mathrm{I}_{0} \mathrm{~T}_{5}$ & $6.2 \mathrm{abcdef}$ & $1.27 \mathrm{a}$ & 0.088 & 32.72 & 0.037 & $37.75 \mathrm{abc}$ \\
$\mathrm{I}_{0} \mathrm{~T}_{6}$ & $6.4 \mathrm{abc}$ & $1.15 \mathrm{f}$ & 0.074 & 30.61 & 0.023 & $14.89 \mathrm{~d}$ \\
$\mathrm{I}_{0} \mathrm{~T}_{7}$ & $6.0 \mathrm{bcdef}$ & $1.17 \mathrm{e}$ & 0.065 & 30.81 & 0.044 & $31.88 \mathrm{bcd}$ \\
$\mathrm{I}_{1} \mathrm{~T}_{0}$ & $5.9 \mathrm{ef}$ & $0.99 \mathrm{l}$ & 0.057 & 20.37 & 0.019 & $21.27 \mathrm{~cd}$ \\
$\mathrm{I}_{1} \mathrm{~T}_{1}$ & $6.3 \mathrm{abcde}$ & $1.05 \mathrm{i}$ & 0.118 & 26.50 & 0.033 & $31.65 \mathrm{bcd}$ \\
$\mathrm{I}_{1} \mathrm{~T}_{2}$ & $6.2 \mathrm{abcdef}$ & $1.12 \mathrm{~h}$ & 0.121 & 33.91 & 0.042 & $29.56 \mathrm{bcd}$ \\
$\mathrm{I}_{1} \mathrm{~T}_{3}$ & $5.9 \mathrm{f}$ & $1.16 \mathrm{f}$ & 0.078 & 30.22 & 0.037 & $54.97 \mathrm{a}$ \\
$\mathrm{I}_{1} \mathrm{~T}_{4}$ & $6.0 \mathrm{def}$ & $1.19 \mathrm{c}$ & 0.057 & 34.77 & 0.044 & $40.09 \mathrm{abc}$ \\
$\mathrm{I}_{1} \mathrm{~T}_{5}$ & $6.4 \mathrm{abc}$ & $1.22 \mathrm{~b}$ & 0.091 & 35.44 & 0.047 & $43.75 \mathrm{ab}$ \\
$\mathrm{I}_{1} \mathrm{~T}_{6}$ & $5.9 \mathrm{f}$ & $1.17 \mathrm{e}$ & 0.069 & 27.97 & 0.030 & $40.85 \mathrm{abc}$ \\
$\mathrm{I}_{1} \mathrm{~T}_{7}$ & $6.0 \mathrm{cdef}$ & $1.18 \mathrm{~d}$ & 0.091 & 30.18 & 0.025 & $26.72 \mathrm{bcd}$ \\
\hline $\mathrm{SE}( \pm)$ & 0.1087 & 0.0127 & $\mathrm{NS}$ & $\mathrm{NS}$ & $\mathrm{NS}$ & 5.434 \\
\hline
\end{tabular}

In a column figures having same letter do not differ significantly whereas figures with different letter differ significantly by DMRT at $5 \%$ level.

$\left[\mathrm{I}_{0}=\right.$ Alternate wetting and drying, $\mathrm{I}_{1}=$ Continuous flooding, $\mathrm{T}_{0}$ : control, $\mathrm{T}_{1}: 100 \% \mathrm{RDCF},\left(\mathrm{N}_{100} \mathrm{P}_{15} \mathrm{~K}_{45} \mathrm{~S}_{20} \mathrm{Zn}_{2}\right), \mathrm{T}_{2}$ : 10 ton cowdung/ha, $\mathrm{T}_{3}: 50 \% \mathrm{RDCF}+5$ ton cowdung/ha, $\mathrm{T}_{4}: 8$ ton poultry manure/ha, $\mathrm{T}_{5}: 50 \% \mathrm{RDCF}+4$ ton poultry manure/ha, $\mathrm{T}_{6}: 10$ ton vermicompost/ha, $\mathrm{T}_{7}: 50 \% \mathrm{RDCF}+5$ ton vermicompost/ha] 


\subsection{Interaction effect of fertilizer and irrigation on $\mathrm{pH}, \mathrm{OM}, \mathrm{N} P \mathrm{~K}$ and $\mathrm{S}$ of post harvest soil}

The combined effect of different sources of fertilizer and irrigation significantly influenced the $\mathrm{pH}, \mathrm{OM}$ and $\mathrm{S}$ content of post harvest soil (Table 5). The highest $\mathrm{pH}$ of post harvest soil (6.5) was recorded with the treatment combination $\mathrm{I}_{0} \mathrm{~T}_{1}$ (Alternate wetting and drying + $100 \%$ RDCF, $\left(\mathrm{N}_{100} \mathrm{P}_{15} \mathrm{~K}_{45} \mathrm{~S}_{20} \mathrm{Zn}_{2}\right)$ ) which was followed (6.4) by $\mathrm{I}_{0} \mathrm{~T}_{0}$ (Alternate wetting and drying + control treatment). On the other hand, the lowest $\mathrm{pH}$ of post harvest soil (5.9) was recorded with the treatment combination $\mathrm{I}_{1} \mathrm{~T}_{3}$ (Continuous flooding $+50 \%$ RDCF plus 5 ton cowdung/ha).

The level of organic matter increased in the post harvest soil due to application of chemical fertilizer plus manure. The highest organic matter of post harvest soil $(1.27 \%)$ was recorded with the treatment combination $\mathrm{I}_{0} \mathrm{~T}_{5}$ (Alternate wetting and drying $+50 \% \mathrm{RDCF}+4$ ton poultry manure/ha) which was followed $(1.22 \%)$ by $\mathrm{I}_{1} \mathrm{~T}_{5}$ (Continuous flooding $+50 \% \mathrm{RDCF}+4$ ton poultry manure/ha). On the other hand, the lowest organic matter of post harvest soil $(0.99 \%)$ was recorded with the treatment combination $\mathrm{I}_{1} \mathrm{~T}_{0}$ (Continuous flooding + control treatment). The $\mathrm{N}, \mathrm{P}$ and $\mathrm{K}$ concentrations in post harvest soils were not significantly affected by combined application of fertilizer and irrigation.

The highest available sulphur in post harvest soil (54.97 ppm) was recorded from $\mathrm{I}_{1} \mathrm{~T}_{3}$ (Continuous flooding $+50 \%$ RDCF +5 ton cow dung/ha). On the other hand, the lowest available sulphur in post harvest soil (14.89 $\mathrm{ppm}$ ) was obtained from $\mathrm{I}_{0} \mathrm{~T}_{6}$ (Alternate wetting and drying +10 ton Vermicompost/ha).

\section{Conclusions}

The integrated use of manure and inorganic fertilizer with different levels of irrigation significantly influenced the yield and yield contributing characters of boro rice. The higher values of yield parameters and yields were recorded in the treatments where fertilizer plus manure were used. The highest effective tillers/hill, plant height, panicle length, 1000 grain wt., grain and straw yields were found from $\mathrm{T}_{5}$ treatment where $50 \%$ RDCF plus 4 ton poultry manure was used. On the other hand, lowest values were obtained from $\mathrm{T}_{0}$ treatment. The number of effective tillers/hill, grain yield and straw yield were significantly influenced by combined application of irrigation and fertilizer. The highest grain and straw yields were recorded from $\mathrm{I}_{0} \mathrm{~T}_{5}$ and the lowest were observed from $\mathrm{I}_{0} \mathrm{~T}_{0}$ treatment combination. The grain and straw nutrient concentration in boro rice plant was significantly influenced by fertilizer and manure. The almost similar levels of $\mathrm{N}, \mathrm{P}, \mathrm{K}, \mathrm{S}$ concentration were recorded from $\mathrm{I}_{0}$ and $\mathrm{I}_{1}$ treatments. The highest concentrations of $\mathrm{N}, \mathrm{P}$, $\mathrm{K}, \mathrm{S}$ in grain and straw were recorded from $\mathrm{T}_{5}$ treatment and in all cases lowest value was observed in $\mathrm{T}_{0}$ treatment. The $\mathrm{pH}$, organic matter and levels of $\mathrm{N}, \mathrm{P}, \mathrm{K}, \mathrm{S}$ of post harvest soil were significantly affected by fertilizer and combined application of irrigation and fertilizer. The higher levels of organic matter and nutrient concentrations were found in the fertilizer treatments where manure plus inorganic fertilizers were used. The highest $\mathrm{pH}$ (6.4) was recorded from $\mathrm{T}_{1}$ and highest organic matter (1.24\%), P (34.08 ppm), K (0.042 m.eq/100g), S (40.76 ppm) were recorded from $\mathrm{T}_{5}$. On the other hand, the lowest values were recorded from $\mathrm{T}_{0}$ treatment.

From the above discussion it can be concluded that irrigation had no significant effect on yield and yield contributing characters and application alternate wetting and drying is preferable than flood irrigation and application of 50\% RDCF + 4 ton poultry manure/ha and alternate wetting and was most favorable for improving yield and yield contributing characters of BRRI dhan 28 in Boro season.

\section{Acknowledgment}

Funding from the Ministry of Science and Technology, Bangladesh for this work is gratefully acknowledged. 


\section{References}

Ayoola, O. T. and Makinde, E. A. 2009. Maize growth, yield and soil nutrient changes with $\mathrm{N}$ enriched organic fertilizers. African Journal of food, Agriculture, Nutrition and Development, 9 (1): 580592.

BBS (Bangladesh Bureau of Statistics). 2008. Statistical Yearbook of Bangladesh. Statistics Division, Ministry of Planning, Govt. of Peoples Republic of Bangladesh. Dhaka. Bangladesh. 71 pp.

FAO (Food and Agricultural Organization). 1999. Yearbook of Production, FAO Statistics Division. 605-607 pp.

Gomez, K. A. and Gomez, A. A. 1984. Statistical Procedures of Agricultural Research, $2^{\text {nd }}$ end., John Wiley and Sons, Singapore, $21 \mathrm{p}$.

Hunter, A. H. 1984. Soil Fertility Analytical Service in Bangladesh. Consultancy Report BARC, Dhaka.

Lin, Xiangin, Zhu, D. and Xinjunlin. 2011. Effects of water management and organic fertilization with SRI crop practices on hybrid rice performance and rhizosphere dynamics. Paddy and Water Environment, 9: 33-39.

Miah, M. A. M., Ishaque, M. and Saha, P. K. 2006. Integrated nutrient management for improving soil health and rice production. Proc. of twenty first BRRI-DAE joint workshops on bridging the rice yield gap for food security. BRRI, Gazipur, Bangladesh, 19-21 September, paper 11: 1-15.
Nayak, D. R., Babu, X. and Adhya, T. K. 2007. Long-term application of compost influences mineral biomass and enzyme activities in a tropical Aeric Endoaquept planted to rice under flooded condition. Soil Microbiology \& Biochemistry, 39 (8): 1897-1906.

Olsen, S. R., Cole, C. V., Watanabe, F. S. and Dean, L. A. 1954. Estimation of available phosphorus in soils by extraction with sodium bicarbonate, U.S. Dept. Agric. Circ., 929 p.

Rahman, M. S., Islam, M. R., Rahman, M. M. and Hossain, M. I. 2009. Effect of cowdung, poultry manure and urea-N on the yield and nutrient uptake of BRRI dhan 29. Bangladesh Research Publications Journal, 2: 552-558.

Singh, R. Singh, S. and Prasad, K. 2001. Effect of fertilizer, FYM and row spacing on transplanted rice. Crop Research, 22 (2): 296-299.

Xu, M. G., Li, D. C., Li, J. M., Qin, D. Z., Yagikazuyuki and Hosen, Y. 2008. Effects of organic manure application with chemical fertilizers or Nutrient Absorption and yield of Rice in Hunan of Southern China. Agricultural Sciences in China, 7 (10): 1245-1252.

Yang, C. M., Yang, L., Yang, Y. and Ouyang, Z. 2004. Rice root growth and nutrient uptake as influenced by organic manure in continuously and alternately flooded paddy soils. Agricultural Water Management, 70 (1): 67-81. 